\title{
Search for Heavy Resonances at the LHC
}

\author{
Sabine Crépé-Renaudin* \\ on behalf of the ATLAS and CMS collaborations \\ Laboratoire de Physique Subatomique et de Cosmologie, Université Grenoble-Alpes, \\ CNRS/IN2P3, Grenoble, France \\ E-mail: crepeein2p3.fr
}

\begin{abstract}
Numerous models beyond the Standard Model theory predict new heavy particles or high energy phenomena that would appear as heavy resonances in collider data. Such signatures were actively searched for by the ATLAS and CMS collaborations using the LHC proton-proton collisions. The most recent analyses using the full dataset $\left(\sim 20 \mathrm{fb}^{-1}\right)$ with center-of-mass collisions at $8 \mathrm{TeV}$ and probing a large variety of final states are reported in this article. No sign of new physics was discovered and the results are interpreted as exclusion limits in a model independent way or in various models based on the presence of extra-dimensions, a new strong sector or large symmetry groups.
\end{abstract}

Frontiers of Fundamental Physics 14 - FFP14,

15-18 July 2014

Aix Marseille University (AMU) Saint-Charles Campus, Marseille

${ }^{*}$ Speaker. 


\section{Introduction}

The Standard Model (SM) is in very good agreement with experimental data. The recent discovery of a Higgs boson by the ATLAS and CMS collaborations brought up the last missing particle of this model. However important features like the gravitational interaction, the dark matter and the origin of the neutrino mass are missing in that theory. It gives also no answer to questions like: why are there three generations of fermions with an important mass hierarchy? Where does the asymmetry between matter and antimatter come from? Why is the Higgs light and the gravity so weak? Models beyond the SM are proposed to solve one or several of these issues. The ones [1] used in the studies that will be presented here, can be classified in three main categories: 1) models assuming compactified extra-dimensions (these extra-dimensions can be flat and large like in the ADD model ${ }^{1}$, or at the TeV scale like in the Universal Extra Dimension model (UED), or at the TeV scale but warped like in the Randall-Sundrum models (RS1 or bulk RS)); 2) models assuming a new strong sector like the Technicolor, little Higgs models or compositness models and 3) models based on larger symmetry groups. The first class of models gives an answer to the gravity weakness, the second addresses electroweak symmetry breaking, naturalness of the Higgs mass and the fermion generations and the third addresses gauge unification or restore the left-right symmetry.

These models lead to various signatures at the LHC. Extra-dimension models imply the production of Kalusa-Klein excitations of particles or strings and black holes ${ }^{2}$. In the case of the second class of models, excited quarks and leptons or leptoquarks may be produced. Finally if there is a larger symmetry group than the SM one, new gauge bosons would appear like the $Z_{\psi}$ or $Z_{\chi}$ in models based on E6 gauge group or a $Z^{\prime}$ boson from the Sequential Standard Model (SSM) or Extended Gauge Model (EGM), with the same couplings to fermions as the usual SM $Z$ boson.

These models predict mostly new heavy particles with a short lifetime that will appear as resonances. In the analyses, these resonances will show up as a Breit-Wigner function peak above a smooth background. However, the resonance peak will be distorted by the parton luminosity distributions and by potential interference effects. This has to be taken into account as well as potential deformation from detector or reconstruction effects.

ATLAS [2] and CMS [3] experiments are powerful tools to pursue this kind of searches. Both collaborations released a large amount of results using the first LHC data taking period that allowed to study about 5 and $20 \mathrm{fb}^{-1}$ of proton-proton collisions at respectively 7 and $8 \mathrm{TeV}$. These proceedings report only on the latest results of these experiments using the full $8 \mathrm{TeV}$ dataset recorded in 2012 and focuses moreover only on singly produced resonances.

\section{Analysis strategy and outline}

When searching for new physics, one strategy consists of studying as many signatures as possible in the most model-independent way. The analyses generally probe highest available energies but also the full available energy range to be sensitive to low cross-section processes. For each final state, analyses search for a deviation with respect to the SM distributions. In case no deviation with respect to the SM prediction is observed, limits are set on the parameters of several available

\footnotetext{
${ }^{1}$ proposed by Arkani-Hamed, Dimopoulos and Dvali

${ }^{2}$ provided that the energy in the collision is above the fundamental higher dimensional Planck scale
} 
models predicting resonance decays in the studied channel. In general, an upper limit on the crosssection times branching fraction is derived, translated subsequently into a lower limit on the new particle mass. In the following, all limits are given at $95 \%$ of confidence level.

The challenges in this kind of analysis are often to measure precisely high energetic particles and to evaluate the uncertainties at high energy as well as to be able to cope with highly boosted topologies.

The analyses described in the following probe dilepton, diquark and diboson final states as well as a mix of those particles.

\section{Lepton final states}

The dilepton final state is generally one of the first to be studied as it benefits from a clear signal signature and a relatively low background. The main challenge is the transverse momentum resolution of the leptons.

In the case where the two leptons are electrons or muons, both ATLAS [4] and CMS [5] select two well isolated leptons. In the electron case, no charge identification is required. Main backgrounds originate from Drell-Yan, diboson, top and jet productions. Misidentified jets are an important background in the electron final states for which the fake rate is measured on data. Examples of dilepton invariant mass distribution are shown in Fig. 3. A good agreement between
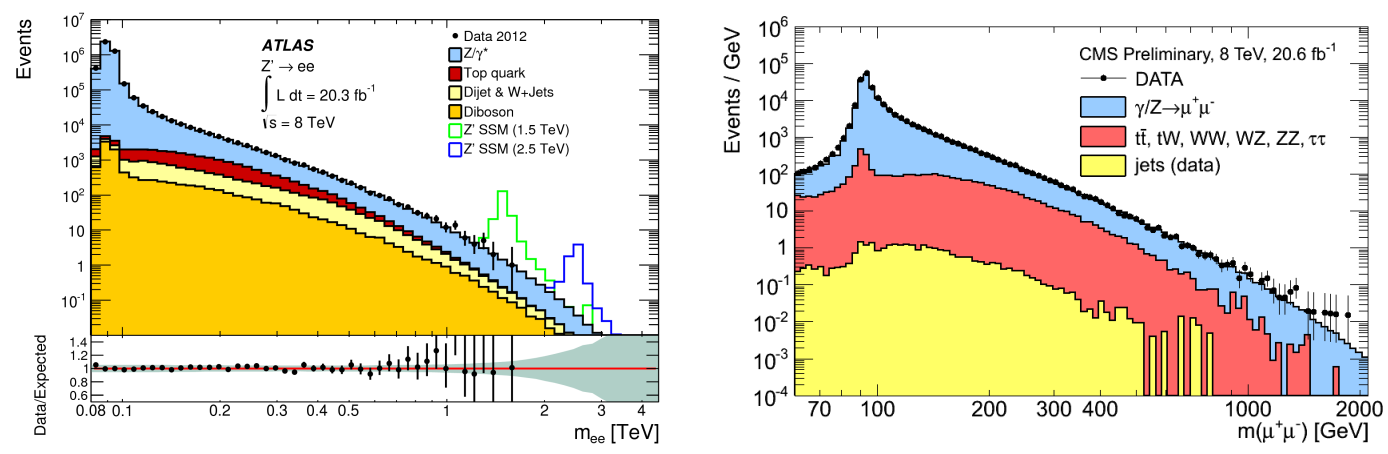

Figure 1: ATLAS dielectron [4] (left) and CMS dimuon [5] invariant mass distributions (right).

data and SM model predictions is found in all channels.

Thus ATLAS and CMS set limits on a SSM $Z^{\prime}$ as well as on an E6 $Z_{\psi}$ or $Z_{\chi}$. ATLAS results are also interpreted in terms of a $Z^{*}$ from a $\left(Z^{*}, W^{*}\right)$ doublet, a spin 2 RS graviton excitation (improperly quoted in the following as "RS graviton"). The limits are in the 1 to $2.9 \mathrm{TeV}$ range and a $Z_{S S M}^{\prime}$ is excluded below respectively 2.96 and $2.90 \mathrm{TeV}$ by CMS and ATLAS.

$W^{\prime}$-like particles may also be searched for with decays in the electron or muon plus neutrino channel. Main backgrounds arise from SM $W, Z$, top, diboson or multijet SM production. ATLAS [6] and CMS [7] set a limit on a SSM $W^{\prime}$ (interference effect are not taken into account in the ATLAS analysis) at 3.2 TeV. Limits in the 1.7-3.2 TeV range are also obtained on an excited chiral $W^{*}$ by ATLAS and on a right handed $W$ and a $W_{K K}^{2}$ from split UED by CMS. The Parton Distribution Functions (PDF) are the main source of uncertainties for these analyses. 
Examples of limits obtained in leptonic channels are given in Fig. 2.
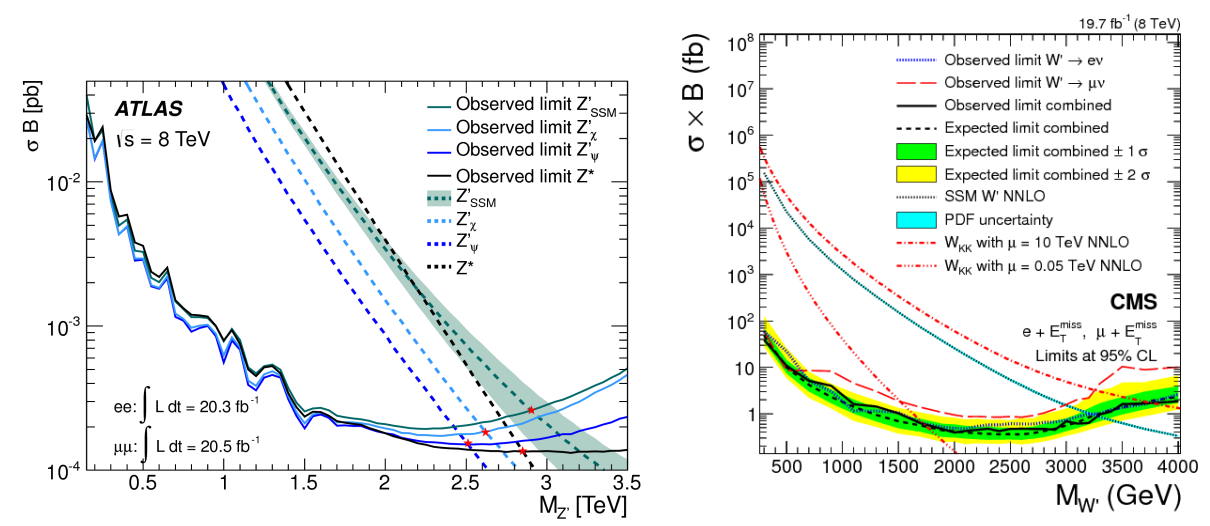

Figure 2: Observed cross-section times branching ratio upper limits for various models, for ATLAS in the dilepton final state [4] (left) and for CMS in the lepton plus neutrino channel [7] (right).

\section{Quark final states}

\subsection{Top-bottom final state}

The search for a $W^{\prime}$ boson in the top+bottom channel completes the leptonic searches. It allows indeed to test models with enhanced couplings to third generation quarks and gives results valid for right handed $W$ even in the case of a right handed neutrino mass higher than a few GeV. CMS performed a search in the semi-leptonic channel [9] whereas ATLAS analyzed the fully hadronic signature [8]. The CMS study is based on the selection of an isolated electron or muon with missing transverse energy and at least two jets. The main backgrounds are ditop, $W$ and $Z$ plus jets, single top and diboson production. In the ATLAS case, as the top from the $W^{\prime}$ decay is boosted, the selection consists of a b-jet and a large radius top jet, in which all particles from the top decay are collimated.

The hadronic analysis is as expected less sensitive than the semi-leptonic one. ATLAS sets an upper limit on the cross-section times branching ratio of the production of a right handed $W\left(W_{R}\right)$ or a left handed one $\left(W_{L}\right)$ (with same quark coupling than the SM $W$ ) respectively in the 0.09-0.21 and $0.14-0.34 \mathrm{pb}$ range for a $W$ mass between 155 and $3000 \mathrm{GeV}$. CMS obtains a lower limit on the $W_{L}$ mass at $2.05 \mathrm{TeV}$ without any interference effect, it decreases to $1.84 \mathrm{TeV}$ when interference is included. This analysis also excludes a $W_{R}$ lighter than $2.05 \mathrm{TeV}$. This limit becomes $2.13 \mathrm{TeV}$ when the right handed neutrino is heavier than the $W_{R}$. CMS also interprets the limits as a function of the left-handed and right-handed coupling strength.

\subsection{Interlude: large jets and boson/top tagging}

A highly massive particle will decay in much lighter SM ones (like a top or a $W$ or $Z$ boson) that will be thus very boosted. The decay products of these boosted particles tend then to be collimated: leptons are no more isolated, jets from quarks tend to merge.

New techniques have been developed to deal with such configurations. The isolation is no more defined with a fixed radius cone but rather with a cone radius that decreases with increasing 

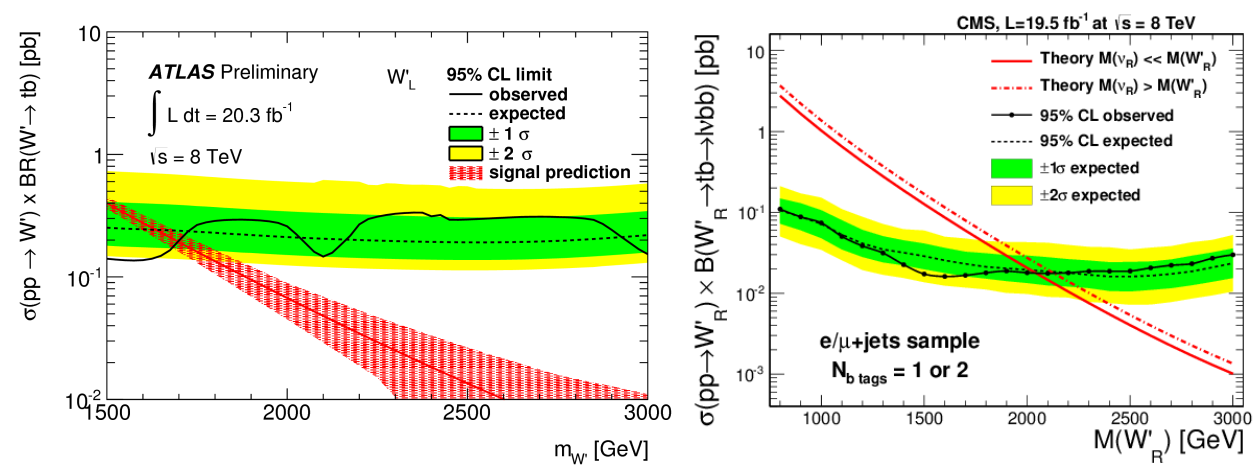

Figure 3: Observed upper cross-section times branching ratio limit for a $W^{\prime}$ to $t b$ decay for ATLAS with a left handed $W^{\prime}$ in the all hadronic final state [8] (left) and for CMS for a right handed $W^{\prime}$ to $t b$ in the semi-leptonic channel [9] (right).

transverse momentum. Large jets are defined using grooming techniques that allow to clean up soft components and pile-up. New variables are built $\left(k_{T}\right.$ splitting scale, $\mathrm{N}$-subjettiness,...) to be sensitive to the jet substructure and tag a 2 or 3 prong $Z / W$ boson or top.

These variables have been successfully used in most of the analyses described here with hadronic final states allowing an efficient selection of boosted signals.

\subsection{Dijet final state}

The dijet final state is challenging since the massive object that couples to quarks and gluons has to be found in the huge multijet QCD background. The trigger plays an important role and ATLAS and CMS differ in their strategy: ATLAS [10] chose a single jet pre-scaled trigger to be sensitive at low mass (with a lower threshold as low as $59 \mathrm{GeV}$ ) whereas CMS [11] used jets from their particle flow algorithm with a $750 \mathrm{GeV}$ lower cut on the dijet mass. Both ATLAS and CMS analyses rely on data to evaluate the background. Main uncertainties are the jet energy scale and resolution and the luminosity measurement. A similar study was performed by CMS with one or two jets tagged as coming from b quarks [12]. Results are given in a model independent way. In addition, limits are derived in the 1.2-6 TeV range for specific models producing excited quarks $\left(q^{*}\right.$, $\left.b^{*}\right)$, strings, new vector bosons $V^{\prime}\left(Z^{\prime}\right.$ or $\left.W^{\prime}\right), W^{*}$, RS graviton or quantum black holes. Figure 4 shows the display of a $5.15 \mathrm{TeV}$ CMS dijet event and one of the limit from ATLAS on the crosssection times branching ratio and acceptance product. Very high mass limits are obtained there: ATLAS ruled out a $q^{*}$ below 4.09 TeV and CMS a quantum black hole below $5.82 \mathrm{TeV}$.

\section{Diboson final states}

\subsection{Vector boson final states : $W W, Z Z, W Z$}

Diboson analyses were performed in all the possible final states. The recent studies reported here are the CMS all jets analysis [13], the lepton plus jets [14, 15] and all leptons [16, 17] ones from both ATLAS and CMS respectively. When jets are present in the final state, boosted techniques are used. The all jets analysis follows a similar scheme than the previously described dijet 

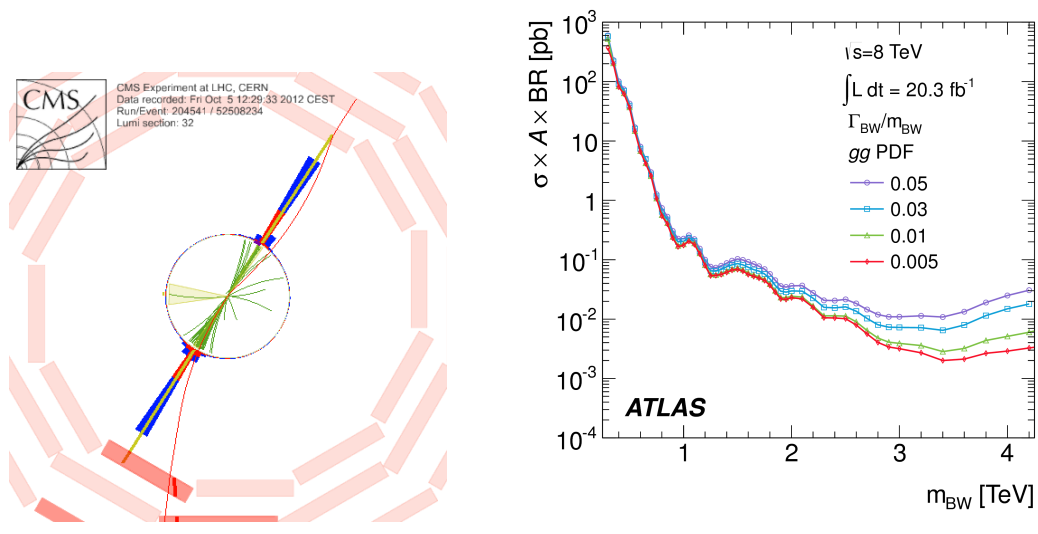

Figure 4: CMS dijet event display with a dijet invariant mass of $5.15 \mathrm{TeV}$ [11] (left) and generic limits from ATLAS on the cross-section times acceptance product for a Breit-Wigner narrow resonance produced by a gg initial state as a function of the mean Breit-Wigner mass [10] (right).

one. It's sensitive to a new particle decaying to two vector bosons $V V$ or a quark and a $V$. The main uncertainties lie on the signal modelling, the boson tagging, the PDF and the jet energy resolution. Limits in the 1.3-3.2 TeV range are derived for excited quark, RS graviton, and $W^{\prime}$.

In the lepton plus jet channel, ATLAS looks for two small radius jets (radius parameter $R_{j e t}=$ $0.4)$ or one big jet $\left(R_{\text {jet }}=1.2\right)$ when CMS uses only jets with $R_{\text {jet }}$ equal to 0.8 . The ATLAS splitting allows to keep a good sensitivity at low mass. Both collaborations use boosted techniques to tag the hadronically decaying boson. CMS deduced model independent limits on event number as a function of the reconstructed $V$ efficiency and combined these with the fully hadronic channel. ATLAS analysis set a limit on an EGM $W^{\prime}$ at $1.59 \mathrm{TeV}$ and a RS bulk $G^{*}$ at $740 \mathrm{GeV}$ for $\frac{k}{\bar{M}_{P l}}=1.0$.

The fully leptonic channel allows to search for a new resonance decaying in $W Z$. It shows a better sensitivity at low mass thanks to its higher mass resolution and its smaller backgrounds that come mainly from SM diboson production as well as top-antitop associated with a $W$ or a $Z$. The main uncertainties are the PDF and the normalisation and factorisation scales for the backgrounds. A good agreement is found with the SM prediction thus ATLAS excluded an EGM $Z^{\prime}$ below $1.5 \mathrm{TeV}$ [16] and CMS a $W^{\prime}$ between 170 and $1450 \mathrm{GeV}$ [17].

\subsection{Higgs-Higgs final state}

ATLAS searched for the first KK excitation of the bulk RS graviton $G^{*}$ that decays in two Higgs where each of the two bosons decays in a b quark pair [18]. The main backgrounds are multijet and top pair production. Dijet that are b-tagged and near the Higgs mass are selected and a top-pair veto is used. The multijet background is controlled using data. A good agreement is found with SM expectations and a graviton mass between 590 and $710 \mathrm{GeV}$ is ruled out for $\frac{k}{\overline{M_{P l}}}=1.0$.

\section{Mixed final states}

Finally, excited quarks are searched for in the photon and jet final state. This s-channel process results in to an isotropic distribution of the particles whereas SM gamma plus jet and multijet backgrounds tend to peak in the forward region. Backgrounds are parametrized using data, like for 
the previous dijet analyses. ATLAS and CMS $[19,20]$ set limits on an excited spin $1 / 2$ quark of first generation at 3.5 and 3.2 TeV respectively. ATLAS also added a limit on an ADD non-thermal quantum black hole at $4.6 \mathrm{TeV}$.

\section{Conclusion and outlook}

ATLAS and CMS have started to explore the TeV scale in a wide range of final states and published beautiful results that contained however no sign of new physics. Only a few analyses have been briefly presented here and there are still many to come: all the results and summary graphs are referenced on the ATLAS [21] and CMS [22] web pages.

Moreover, the $13 \mathrm{TeV}$ LHC run will allow to probe a higher mass range taking advantage of the 10 to 100 higher parton luminosity. Lower mass ranges will however not be neglected since it allows to be sensitive to small couplings with the expected $300 \mathrm{fb}^{-1}$ of integrated luminosity. Besides, techniques to deal with high energy, high luminosity and boosted topologies show constant progress that will allow to look efficiently for new signals. The LHC run 2 will thus open the door to test new models, to be more sensitive using combinations of channel and to develop analyses in a more model-independent way. New physics is perhaps just around the corner !

\section{References}

[1] It was not possible, in the scope of this proceeding, to add all the needed theory references used by the experimental studies summarized here. These references may be found in each of the experimental ones below.

[2] ATLAS Collaboration, JINST 3 (2008) S08003.

[3] CMS Collaboration, JINST 3 (2008) S08004.

[4] ATLAS Collaboration, Phys. Rev. D 90 (2014) 052005, [arXiv:1405.4123].

[5] CMS Collaboration, CMS-PAS-EXO-12-061, http://cds.cern.ch/record/1519132.

[6] ATLAS Collaboration, ATLAS-CONF-2014, http://cds.cern.ch/record/1562841.

[7] CMS Collaboration, Submitted to Phys. Rev. D, [arXiv:1408.2745].

[8] ATLAS Collaboration, submitted to Eur. Phys. J. C, [arXiv:1408.0886].

[9] CMS Collaboration, JHEP 05 (2014) 108, [arXiv:1402.2176].

[10] ATLAS Collaboration, submitted to Phys. Rev. D, [arXiv:1407.1376].

[11] CMS Collaboration, CMS-PAS-EXO-2012-059, http://cds.cern.ch/record/1519066.

[12] CMS Collaboration, CMS-PAS-EXO-12-023, http://cds.cern.ch/record/1542405.

[13] CMS Collaboration, JHEP 08 (2014) 173, [arXiv:1405.1994].

[14] ATLAS Collaboration, ATLAS-COM-CONF-2014-039, http://cds.cern.ch/record/1735253.

[15] CMS Collaboration, JHEP 08 (2014) 174, [arXiv:1405.3447].

[16] ATLAS Collaboration, Phys. Lett. B 737 (2014) 174, [arXiv:1406.4456].

[17] CMS Collaboration, Phys. Lett. B 740 (2015) 83, [arXiv:1407.3476].

[18] ATLAS Collaboration, ATLAS-CONF-2014-005, http://cds.cern.ch/record/1666518.

[19] ATLAS Collaboration, Phys. Lett. B 728 (2014) 562, [arXiv:1309.3230].

[20] CMS Collaboration, Phys. Lett. B 738 (2014) 274, [arXiv:1406.5171].

[21] https://twiki.cern.ch/twiki/bin/view/AtlasPublic/ExoticsPublicResults .

[22] https://twiki.cern.ch/twiki/bin/view/CMSPublic/PhysicsResultsEXO https://twiki.cern.ch/twiki/bin/view/CMSPublic/PhysicsResultsB2G . 